\title{
Vav3 oncogene is upregulated and a poor prognostic factor in breast cancer patients
}

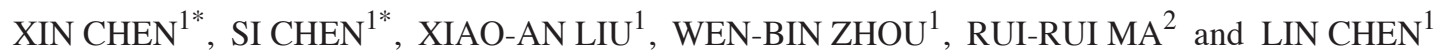 \\ ${ }^{1}$ Department of Breast Surgery, First Affiliated Hospital of Nanjing Medical University, Nanjing, Jiangsu 210029; \\ ${ }^{2}$ Department of General Surgery, Songjiang Central Hospital, Shanghai 201699, P.R. China
}

Received April 27, 2014; Accepted January 29, 2015

DOI: $10.3892 / \mathrm{ol} .2015 .3004$

\begin{abstract}
The Vav3 oncogene is overexpressed and has a significant role in the tumorigenesis of prostate cancer and glioblastoma. In the present study, the expression status and prognostic value of Vav3 expression was investigated in breast cancer. Vav3 protein levels were analyzed by immunoblotting in human breast cancer and epithelial cell lines. Immunohistochemistry was used to detect Vav3 in a tissue microarray of 173 breast cancers and 19 benign breast lesions. Statistical analysis was performed to reveal the association between Vav3 expression and clinicopathological parameters. Disease-free survival (DFS) and overall survival (OS) were calculated by Kaplan-Meier analysis and the Cox regression model. The Vav3 protein level was higher in the breast cancer cell lines than in the normal human breast cells. Vav3 was expressed in $86.1 \%$ of breast cancer patients, but in only $15.6 \%$ patients with benign breast disease. Patients with negative estrogen receptor expression, axillary lymph node involvement and a high tumor-node-metastasis stage demonstrated a higher positive rate of Vav3 expression. The Kaplan-Meier survival analysis revealed that patients with higher Vav3 expression exhibited shorter DFS and OS times. The multivariate Cox analysis revealed that Vav3 was a prognostic factor of survival. Overall, Vav3 was overexpressed in human breast cancer cells and this correlated with a shorter survival time, indicating that Vav3 is a biomarker of a poor prognosis for breast cancer patients.
\end{abstract}

\section{Introduction}

Breast cancer is the most common malignancy in females (1). Treatments for breast cancer patients include surgery and chemotherapy, as well as radiation, hormone and molecular-targeted therapies, and yet metastasis and recurrence remain clinical

Correspondence to: Dr Lin Chen, Department of Breast Surgery, First Affiliated Hospital of Nanjing Medical University, 300 Guangzhou Road, Nanjing, Jiangsu 210029, P.R. China

E-mail: hxchenlin@aliyun.com

${ }^{*}$ Contributed equally

Key words: Vav3, breast cancer, overexpression, prognosis challenges for a substantial proportion of patients. Biomarkers for breast cancer are urgently required for early diagnosis, patient stratification and prognosis determination.

The Vav proteins are guanine nucleotide exchange factors for GTPases of the Rho family. Vav proteins are involved in cell signaling and tumorigenesis $(2,3)$. The first report of a Vav protein (now known as Vav1) was in 1989, as the result of cell transformation experiments that determined it was a human oncogene (4). Subsequent to this discovery, two more Vav proteins, Vav2 and Vav3, have been identified in mammals (5). Vav2 and Vav3 are expressed in the majority of tissues, while Vav1 is mostly expressed in cells of hematopoietic lineage (6).

The Vav3 oncogene is involved in various cellular signaling processes, including cytoskeleton organization, calcium influx, gene transcription, cell transformation, cell proliferation and apoptosis (2). Vav3 has been found to be overexpressed in human prostate cancer cells and has been proposed to promote the tumorigenesis of prostate cancer $(7,8)$. Vav3 enhances cell growth and proliferation by activating androgen receptor-mediated signaling pathways (8). Breast cancer and prostate cancer are hormone-dependent tumors, whose growth is mediated by their respective hormone receptors. Vav3 is an upstream mediator of Ras-related C3 botulinum toxin substrate 1, which enhances the transcriptional activity of estrogen receptor $\alpha(E R-\alpha)$ in breast cancer cells (9). In addition, Vav3 is epigenetically regulated during the development of breast cancer (10). Thus, it is intriguing to postulate that the progression and maintenance of breast cancer relies on the deregulation of the Vav3 oncogene.

In the present study, the expression of Vav3 in breast cancer and benign breast lesion tissues was analyzed, and the clinical and prognostic significance of Vav3 expression in human breast cancer was evaluated.

\section{Materials and methods}

Cell culture. The human breast cancer cell lines, MCF7 and MDA-MB-231, and tamoxifen-resistant (TAM-R) breast cancer cells were kindly provided by Dr Ping Fan (University of Virginia Health Sciences System, Charlottesville, VA, USA). In addition, non-tumor human breast epithelial MCF10A cells were obtained from the American Type Culture Collection (Rockville, MD, USA). MCF7, MDA-MB-231 and MCF10A cells were cultured in Dulbecco's modified Eagle's medium (Invitrogen, Carlsbad, CA, USA) supplemented with $10 \%$ fetal 
Table I. Clinicopathological characteristics of 173 breast cancer patients.

\begin{tabular}{|c|c|c|}
\hline Characteristic & Number of patients & $\%$ \\
\hline \multicolumn{3}{|l|}{ Age at diagnosis, years } \\
\hline$\leq 35$ & 6 & 3.5 \\
\hline $35-55$ & 100 & 57.8 \\
\hline$>55$ & 67 & 38.7 \\
\hline \multicolumn{3}{|l|}{ Tumor size, cm } \\
\hline$\leq 2$ & 97 & 56.1 \\
\hline $2-5$ & 66 & 38.2 \\
\hline$>5$ & 10 & 5.7 \\
\hline \multicolumn{3}{|l|}{ Lymph node stage } \\
\hline No & 94 & 54.3 \\
\hline N1 & 45 & 26.0 \\
\hline $\mathrm{N} 2$ & 20 & 11.6 \\
\hline N3 & 14 & 8.1 \\
\hline \multicolumn{3}{|l|}{ Histological subtype } \\
\hline Invasive ductal carcinoma & 151 & 87.3 \\
\hline Invasive lobular carcinoma & 12 & 7.0 \\
\hline Ductal carcinoma in situ & 4 & 2.3 \\
\hline Other $^{\mathrm{a}}$ & 6 & 3.4 \\
\hline \multicolumn{3}{|l|}{ Estrogen receptor } \\
\hline Positive & 109 & 63.0 \\
\hline Negative & 64 & 37.0 \\
\hline \multicolumn{3}{|l|}{ Progesterone receptor } \\
\hline Positive & 119 & 68.8 \\
\hline Negative & 54 & 31.2 \\
\hline \multicolumn{3}{|l|}{ HER2 } \\
\hline Positive & 46 & 26.6 \\
\hline Negative & 127 & 73.4 \\
\hline \multicolumn{3}{|l|}{ TNM staging } \\
\hline $\mathrm{I}$ & 62 & 35.8 \\
\hline II & 75 & 43.4 \\
\hline III & 35 & 20.2 \\
\hline IV & 1 & 0.6 \\
\hline
\end{tabular}

${ }^{a}$ Other includes mucinous carcinoma, tubular carcinoma and papillary carcinoma. HER2, human epidermal growth factor receptor 2; TNM, tumor-node-metastasis.

bovine serum (Gibco BRL, Gaithersburg, MD, USA) at $37^{\circ} \mathrm{C}$ in a humidified atmosphere of $5 \% \mathrm{CO}_{2}$. TAM-R cells derived from MCF7 cells were continuously cultured in the above medium containing $10^{-7} \mathrm{~mol} / 1$ tamoxifen.

Western blot analysis. Total proteins from the MCF7, MDA-MB-231, TAM-R and MCF10A cells were extracted on ice with cell lysis buffer (Cell Signaling Technology, Inc., Danvers, MA, USA). Equal amounts of protein were separated by $10 \%$ SDS-PAGE and transferred to a polyvinylidene difluoride membrane. The membranes were blocked with $2 \%$ skimmed milk in phosphate-buffered saline (PBS) at room temperature for $1 \mathrm{~h}$ and probed with the primary polyclonal goat anti-human Vav3 (1:300; cat. no. ab21208; Abcam, Cambridge, MA, USA) and monoclonal mouse anti-human glyceraldehyde-3-phosphate dehydrogenase (1:1,000; cat. no. 60004-1, Proteintech Group, Chicago, IL, USA) antibodies overnight at $4^{\circ} \mathrm{C}$ in PBS containing $0.1 \%$ Tween-20 (PBST) and $1 \%$ skimmed milk. The membranes were then washed four times in PBST and incubated with monoclonal goat anti-rabbit horseradish peroxidase-conjugated secondary antibody (1:1,000; cat. no. STAR54, Bio-Rad Laboratories, Inc., Hercules, CA, USA). Signals were developed with enhanced chemiluminescent reagents (Amersham Pharmacia Biotech, Piscataway, NJ, USA).

Patients and follow-up. This study was approved by the Ethics Committee of the First Affiliated Hospital of Nanjing Medical University (Nanjing, Jiangsu, China). A cohort of 173 breast cancer patients and 19 patients with benign breast disease 
(fibroadenosis or fibroadenoma) were recruited for the study. Written informed consent was obtained from all patients. The patients underwent surgical treatment between the beginning of 2004 and the end of 2007. ER, progesterone receptor (PR) and human epidermal growth factor receptor 2 (HER2) status were determined by immunohistochemistry (Table I).

The patients were followed up every three months for the first two years, every six months for the next three years and once a year after five years. Chest computed tomography, mammography or breast sonography (for patients $\leq 35$ years old), radionuclide bone scans, abdominal sonography, serum tumor marker analysis and detailed physical examinations were routinely performed. No patients were lost to follow-up.

Tissue microarray construction. A tissue microarray from the 192 selected patients was constructed as previously described (11-15). In brief, hematoxylin and eosin-stained sections of the primary tumors were reviewed, and areas of tumors were marked on the slides. Tissue microarrays were constructed by removing $1-\mathrm{mm}$ cores from selected paraffin-fixed tissue blocks and transferring them to a recipient paraffin block using a Manual Tissue Arrayer (Beecher Instruments, Silver Spring, MD, USA). Cores were spaced at intervals of $1.5 \mathrm{~mm}$. All samples were spotted in duplicate, corresponding to the respective areas of the same original paraffin block. Sections of $4-\mu \mathrm{m}$ thick histological cuts were obtained from the tissue microarray and fixed onto glass slides with adhesive film.

Immunohistochemistry. After the 4- $\mu \mathrm{m}$ tissue microarray sections were deparaffinized, endogenous peroxidase activity was blocked with $0.3 \%$ hydrogen peroxide for $10 \mathrm{~min}$, followed by incubation with a polyclonal primary antibody against Vav3 (1:50, Upstate Biotechnology, Lake Placid, NY, USA) for $1 \mathrm{~h}$. Subsequent to washing in PBS three times, sections were incubated for $40 \mathrm{~min}$ with the secondary antibody (BioGenex Laboratories, Inc., San Ramon, CA, USA) at room temperature. After washing, the sections were incubated with streptavidin-conjugated peroxidase (BioGenex).

The intensity and extent of cytoplasm-positive labeling for Vav3 in the tissue arrays were assessed semi-quantitatively and scored as follows: 0, no staining; $1+$, weak and focal staining in $<30 \%$ of the tissues; $2+$, moderate intensity staining in $30-50 \%$ of the tissues; or $3+$, strong and diffuse staining in $>50 \%$ of the tissues. A score of 0 was defined as negative for Vav3 labeling.

For hormone receptors, the staining intensity was scored as follows: Negative, -; low, 1+; moderate, 2+; or strong, 3+. Invasive tumor cell nuclear staining $\geq 10 \%$ was considered hormone receptor-positive, while $<10 \%$ was considered negative. The criteria for positive HER2 was 3+ uniform cell membrane staining in $>30 \%$ of tumor cells. Negative HER2 was 0 , or $1+$ cell surface protein expression in any percentage of staining. HER 2 scored as $2+$ and $3+$ in $<30 \%$ of tumor cells by immunohistochemistry was further confirmed by fluorescence in situ hybridization.

Statistical analyses. Statistical analyses were performed using STATA 10.0 software (StataCorp LP, College Station, TX, USA). The Student's t-test was used to determine the differences in Vav3 expression. Differences in proportions were evaluated with the $\chi^{2}$ or Fisher's exact tests. The Kaplan-Meier method was used

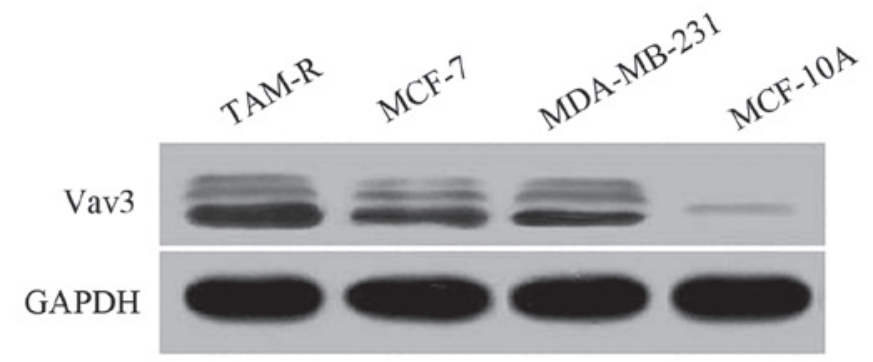

Figure 1. Vav3 is overexpressed in breast cancer cells. The protein levels of Vav3 in MCF-10A, MCF7, MDA-MB-231 and TAM-R cells were determined by western blot analysis. Glyceraldehyde-3-phosphate dehydrogenase served as the loading control.

to calculate the non-parametric survival plots, and the difference was determined by the log-rank test. Disease-free survival (DFS) was calculated as the time from the date of diagnosis to the occurrence of locoregional or distant metastasis. The overall survival (OS) period was calculated from the date of diagnosis to mortality or the date of last follow-up. The Cox regression model was used to evaluate the prognostic significance of Vav3. $\mathrm{P}<0.05$ was considered to indicate a statistically significant difference.

\section{Results}

Vav3 oncogene is overexpressed in human breast cancers. To determine the expression status of Vav3 in breast cancers, the Vav3 protein levels in breast cancer cell lines were first checked using western blot analysis. Compared with the breast epithelial MCF10A cells, the cells of the breast cancer MCF7 and MDA-MB-231 cell lines, and the TAM-R cells revealed an apparently higher expression level of Vav3 (Fig. 1).

To extend this observation in vivo, immunohistochemistry was used to evaluate Vav3 expression in the tissue microarray, which contained 173 human primary breast cancers and 19 benign breast tissues. Vav3 was mainly located in the cytoplasm and nucleus of the epithelial cells of the breast tissues, but not in the stroma (Fig. 2). Vav3 was detected in 3 of 19 (15.8\%) normal breast tissues, including one case of moderate intensity and two cases of weak intensity. In the breast carcinoma tissues, 149 of $173(86.1 \%)$ tumor specimens stained positive for Vav3 $(\mathrm{P}<0.05)$. These in vitro and in vivo data demonstrated that Vav3 was overexpressed in the breast cancer cells.

Correlation between Vav3 expression and clinicopathological features. Next, the clinicopathological features of Vav3-positive and Vav3-negative breast cancers were analyzed (Table II). The expression of Vav3 was significantly correlated with the clinical tumor-node-metastasis (TNM) phase $(\mathrm{P}=0.0309)$, pathological type $(\mathrm{P}=0.007)$, ER status $(\mathrm{P}=0.038)$ and axillary lymph node involvement $(\mathrm{P}=0.045)$. There was no correlation between Vav3 expression and tumor size, HER 2 overexpression, PR status, age at diagnosis or p53 status $(\mathrm{P}>0.05)$.

Prognostic value of Vav3 in breast cancer patients. The median follow-up period was 59 months (range, 46-85 months). The OS rate at the end of the follow-up period was $87.3 \%$. At the end of the follow-up, $140(80.9 \%)$ patients 
Table II. Association of Vav3 expression status with clinicopathological and molecular characteristics.

\begin{tabular}{|c|c|c|c|c|}
\hline Characteristic & Number of patients & Vav3-positive, $\mathrm{n}$ & $\%$ & P-value \\
\hline \multicolumn{5}{|l|}{ Age at diagnosis, years } \\
\hline$\leq 35$ & 6 & 5 & 83.3 & \multirow[t]{2}{*}{0.3860} \\
\hline$>35$ & 167 & 144 & 86.2 & \\
\hline \multicolumn{5}{|l|}{ Tumor size, cm } \\
\hline$\leq 2$ & 97 & 85 & 87.6 & \multirow[t]{3}{*}{0.2680} \\
\hline $2-5$ & 66 & 55 & 83.3 & \\
\hline$>5$ & 10 & 9 & 90.0 & \\
\hline \multicolumn{5}{|l|}{ Lymph node } \\
\hline Negative & 94 & 76 & 80.9 & \multirow[t]{2}{*}{0.0450} \\
\hline Positive & 79 & 73 & 86.7 & \\
\hline \multicolumn{5}{|l|}{ Histological subtype } \\
\hline Invasive ductal carcinoma & 151 & 133 & 88.1 & \multirow[t]{4}{*}{0.0070} \\
\hline Invasive lobular carcinoma & 12 & 11 & 91.7 & \\
\hline Ductal carcinoma in situ & 4 & 1 & 25.0 & \\
\hline Other ${ }^{\mathrm{a}}$ & 6 & 4 & 66.7 & \\
\hline \multicolumn{5}{|l|}{ Estrogen receptor } \\
\hline Positive & 109 & 89 & 69.0 & \multirow[t]{2}{*}{0.0380} \\
\hline Negative & 64 & 60 & 93.8 & \\
\hline \multicolumn{5}{|l|}{ Progesterone receptor } \\
\hline Positive & 119 & 99 & 83.2 & \multirow[t]{2}{*}{0.1530} \\
\hline Negative & 54 & 50 & 92.6 & \\
\hline \multicolumn{5}{|l|}{ HER2 } \\
\hline Positive & 46 & 43 & 93.5 & \multirow[t]{2}{*}{0.1340} \\
\hline Negative & 127 & 106 & 83.5 & \\
\hline \multicolumn{5}{|l|}{ TNM staging } \\
\hline I-II & 137 & 114 & 83.2 & \multirow[t]{2}{*}{0.0309} \\
\hline III-IV & 36 & 35 & 97.2 & \\
\hline
\end{tabular}

${ }^{a}$ Other includes mucinous carcinoma, tubular carcinoma and papillary carcinoma. HER2, human epidermal growth factor receptor 2; TNM, tumor-node-metastasis.

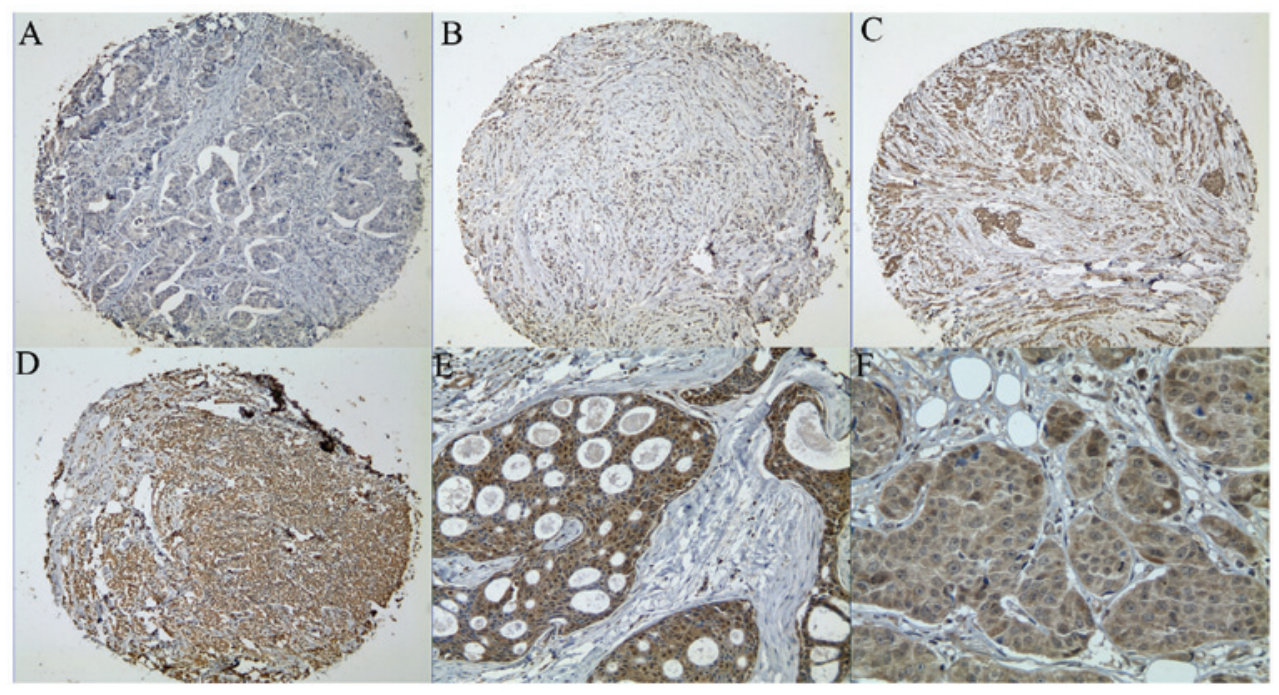

Figure 2. Vav3 is generally upregulated in human primary breast cancer tissues. Vav3 protein levels in human primary breast cancers and benign breast lesions were analyzed using immunohistochemistry. Images shown are representative human primary breast cancer and benign breast lesion specimens from the tissue microarray with (A) negative, (B) weak, (C) moderate and (D) strong positive staining reactions for Vav3. (E and F) Vav3 immunoreactivity was observed in the cytoplasm and nucleus of the epithelial cells, but not in the stroma of breast tissues. 

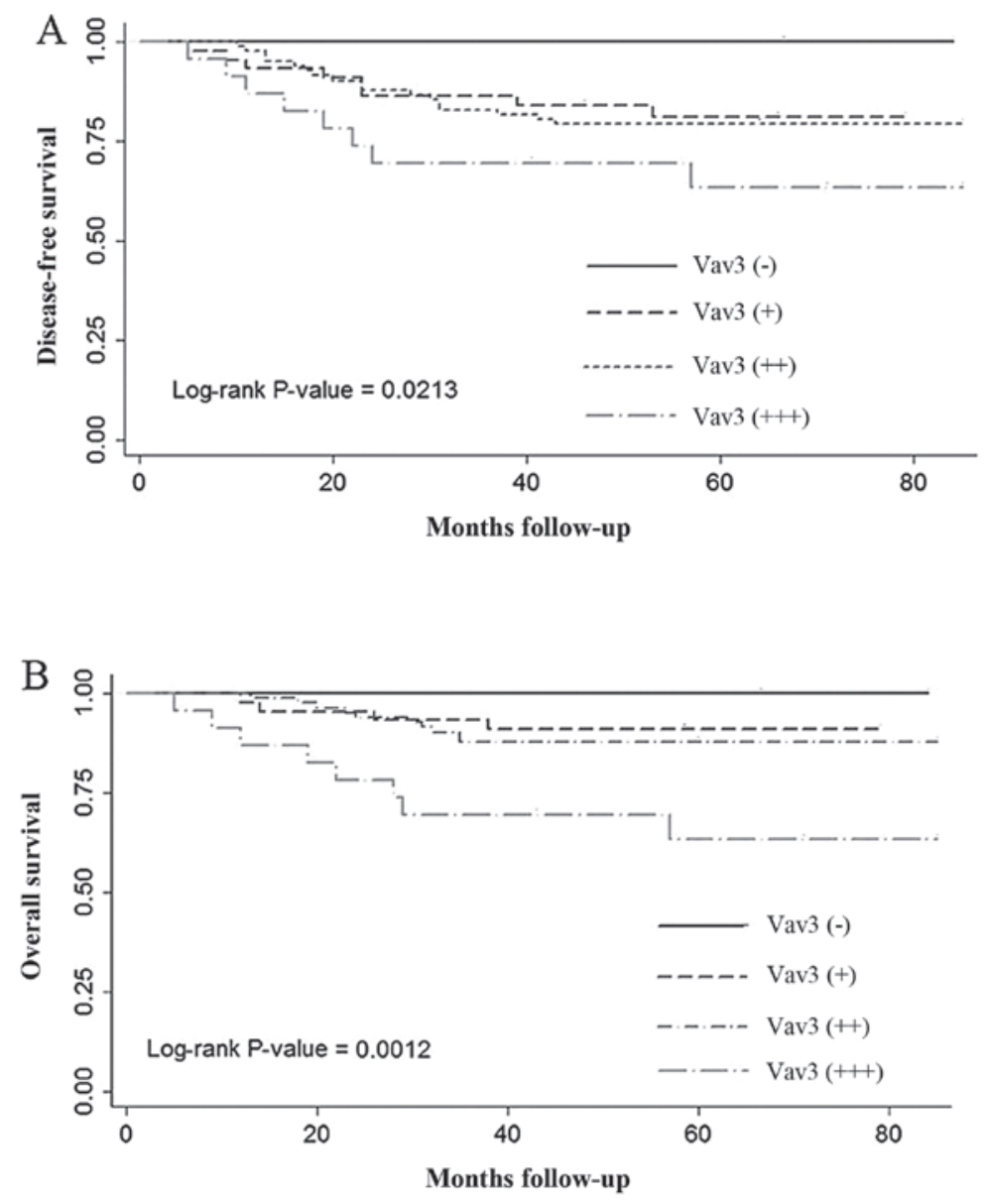

Figure 3. Overexpression of Vav3 is associated with shorter disease-free survival (DFS) and overall survival (OS) in breast cancer patients. Kaplan-Meier curves estimated the DFS and OS with respect to patients with negative, weak, moderate and strong expression of Vav3. (A) Kaplan-Meier analysis of DFS ( $\mathrm{P}=0.0213)$. (B) Kaplan-Meier analysis of OS $(\mathrm{P}=0.0012)$.

were free of disease. Among the $33(19.1 \%)$ patients with events, six presented with local recurrence, 14 with distant metastasis (including 12 mortalities) and three with contralateral metastasis, and 10 succumbed to unknown causes.

When all breast cancer patients were divided into groups based on Vav3 expression (negative, weak, moderate or strong), using the log-rank test, it was observed that patients with strong positive expression of Vav3 experienced the shortest DFS ( $\mathrm{P}=0.0213)$ and OS $(\mathrm{P}=0.0012)$ times (Fig. 3). In the multivariate Cox regression analysis, overexpression of Vav3 was associated with poor DFS $(\mathrm{P}=0.019)$ and OS $(\mathrm{P}=0.004)$ when the age at diagnosis, tumor size, TNM stage and lymph node status were adjusted.

\section{Discussion}

High levels of Vav3 have been observed in various types of cancer, including glioblastoma (16) and prostate cancer (8). In the present study, Vav3 was observed to be significantly upregulated in breast cancers compared with benign breast diseases. Furthermore, Vav3 was identified to be a biomarker of a poor prognosis in breast cancer.

The Vav3 oncogene induces cell transformation (17) and mediates receptor protein tyrosine kinase signaling, including that of the epidermal growth factor, insulin and insulin-like growth factor I receptors. Vav3 suppresses apoptosis by activating the Ras/Raf/MEK/ERK/Elk-1 signaling pathway (18). Furthermore, while neovascularization is inherent to the growth and metastasis of tumors, Vav3 has been found to enhance tumor angiogenesis by stimulating the activation of the EphA2 receptor-mediated signaling pathway, which has a crucial role in the growth of vascular endothelial cells $(19,20)$. Thus, the progression and maintenance of tumors may depend on the deregulation of Vav3.

In the present study, Vav3 protein levels were elevated in the human breast cancer cells in comparison with the human breast epithelial cells. Immunohistochemical analysis revealed that Vav3 was expressed in $86.1 \%$ of breast cancers, but in only $15.8 \%$ of benign breast diseases. Most significantly, a close association was noted between Vav3 expression and several indicators of a poor prognosis in breast cancer, including ER negativity, axillary lymph node involvement and advanced TNM stage.

Previous studies reported that ER was a sensitive prognostic factor and that patients that were ER-negative had poor DFS $(21,22)$. The present study observed that Vav3 expression levels were significantly higher in ER-negative patients. In addition, the Vav3-positive group contained more patients with axillary lymph node involvement, which is also a significant prognostic factor (23), suggesting that patients with increased Vav3 may have a poorer outcome.

In the present study, the expression rate of Vav3 in the patients with stage III-IV breast cancer ( $97.2 \%$ ) was significantly higher 
than that in the patients with stage I-II breast cancer (83.2\%). However, no significant association was noted among the four TNM staging subgroups, possibly due to the small sample size. It was previously reported that patients younger than 35 years frequently presented with high-grade breast cancers, which predicted a poorer outcome in these young patients (24). However, in the present study, Vav3 expression was not associated with age, which may be explained by the small population of patients aged less than 35 years.

Results of the Kaplan-Meier survival analysis demonstrated that patients with the highest expression of Vav3 had the poorest DFS and OS times, and this was further supported by the multivariate analysis. Together, these data suggest that Vav3 is an independent factor in the prognosis of breast cancer.

The current study is limited by the relatively small sample size, which may lead to selection bias. Furthermore, as there is no international standard to define the Vav3 expression level, large population-based studies are required to determine a reference for evaluating Vav3 expression. Finally, the study was based on a retrospective analysis. Prospective studies are required to further investigate Vav3 expression in breast cancer.

In conclusion, the present study demonstrated that Vav3 was upregulated in breast cancer and associated with poor survival, suggesting that Vav3 is a biomarker for the prognosis of breast cancer.

\section{Acknowledgements}

This study was supported by the Priority Academic Program Development of Jiangsu Higher Education Institutions.

\section{References}

1. Parkin DM, Bray F, Ferlay J and Pisani P: Global cancer statistics, 2002. CA Cancer J Clin 55: 74-108, 2005.

2. Bustelo XR: The VAV family of signal transduction molecules. Crit Rev Oncog 7: 65-88, 1996.

3. Van Aelst L and D'Souza-Schorey C: Rho GTPases and signaling networks. Genes Dev 11: 2295-2322, 1997.

4. Katzav S, Martin-Zanca D and Barbacid M: vav, a novel human oncogene derived from a locus ubiquitously expressed in hematopoietic cells. EMBO J 8: 2283-2290, 1989.

5. Zugaza JL, Lopez-Lago MA, Caloca MJ, Dosil M, Movilla N and Bustelo XR: Structural determinants for the biological activity of Vav proteins. J Biol Chem 277: 45377-45392, 2002.

6. Bustelo XR: Regulatory and signaling properties of the Vav family. Mol Cell Biol 20: 1461-1477, 2000.

7. Lyons LS and Burnstein KL: Vav3, a Rho GTPase guanine nucleotide exchange factor, increases during progression to androgen independence in prostate cancer cells and potentiates androgen receptor transcriptional activity. Mol Endocrinol 20: 1061-1072, 2006.
8. Dong Z, Liu Y, Lu S, et al: Vav3 oncogene is overexpressed and regulates cell growth and androgen receptor activity in human prostate cancer. Mol Endocrinol 20: 2315-2325, 2006.

9. Rosenblatt AE, Garcia MI, Lyons L, et al: Inhibition of the Rho GTPase, Rac1, decreases estrogen receptor levels and is a novel therapeutic strategy in breast cancer. Endoc Relat Cancer 18: 207-219, 2011.

10. Loss LA, Sadanandam A, Durinck S, et al: Prediction of epigenetically regulated genes in breast cancer cell lines. BMC Bioinformatics 11: 305, 2010.

11. Sauter G, Simon R and Hillan K: Tissue microarrays in drug discovery. Nat Rev Drug Discov 2: 962-972, 2003.

12. Torhorst J, Bucher C, Kononen J, et al: Tissue microarrays for rapid linking of molecular changes to clinical endpoints. Am J Pathol 159: 2249-2256, 2001.

13. Nocito A, Bubendorf L, Tinner EM, et al: Microarrays of bladder cancer tissue are highly representative of proliferation index and histological grade. J Pathol 194: 349-357, 2001.

14. Moch H, Schraml P, Bubendorf L, et al: High-throughput tissue microarray analysis to evaluate genes uncovered by cDNA microarray screening in renal cell carcinoma. Am J Pathol 154: 981-986, 1999.

15. Kononen J, Bubendorf L, Kallioniemi A, et al: Tissue microarrays for high-throughput molecular profiling of tumor specimens. Nat Med 4: 844-847, 1998.

16. Salhia B, Tran NL, Chan A, et al: The guanine nucleotide exchange factors trio, Ect 2 and Vav3 mediate the invasive behavior of glioblastoma. Am J Pathol 173: 1828-1838, 2008.

17. Zeng L, Sachdev P, Yan L, et al: Vav3 mediates receptor protein tyrosine kinase signaling, regulates GTPase activity, modulates cell morphology and induces cell transformation. Mol Cell Biol 20: 9212-9224, 2000.

18. Palmby TR, Abe K, Karnoub AE and Der CJ: Vav transformation requires activation of multiple GTPases and regulation of gene expression. Mol Cancer Res 2: 702-711, 2004.

19. Hunter SG, Zhuang G, Brantley-Sieders D, Swat W, Cowan CW and Chen J: Essential role of Vav family guanine nucleotide exchange factors in EphA receptor-mediated angiogenesis. Mol Cell Biol 26: 4830-4842, 2006

20. Fang WB, Brantley-Sieders DM, Hwang Y, Ham AJ and Chen J: Identification and functional analysis of phosphorylated tyrosine residues within EphA2 receptor tyrosine kinase. J Biol Chem 283: 16017-16026, 2008.

21. Vollenweider-Zerargui L, Barrelet L, Wong Y, Lemarchand-Beraud T and Gomez F: The predictive value of estrogen and progesterone receptors' concentrations on the clinical behavior of breast cancer in women. Clinical correlation on 547 patients. Cancer 57: 1171-1180, 1986.

22. Viale G, Regan MM, Maiorano E, et al: Prognostic and predictive value of centrally reviewed expression of estrogen and progesterone receptors in a randomized trial comparing letrozole and tamoxifen adjuvant therapy for postmenopausal early breast cancer: BIG 1-98. J Clin Oncol 25: 3846-3852, 2007.

23. Altan E and Altundag K: Clinical and pathological characteristics of occult breast cancer and review of the literature. J BUON 16: 434-436, 2011.

24. Colleoni M, Rotmensz N, Robertson C, et al: Very young women ( $<35$ years) with operable breast cancer: features of disease at presentation. Ann Oncol 13: 273-279, 2002. 